\title{
Quand l'expertise scientifique construit la précaution : le cas des maladies à Prions
}

\section{When scientific expertise shape precaution: the case of Prion diseases}

\section{Marc Barbier* et Céline Granjou**}

* Marc BARBIER est Chargé de recherche à l’Institut National de Recherche Agronomique. Chercheur en gestion des organisations, il travaille à une compréhension des transformations du monde agricole à travers les changements importants que vit le système de recherche et de développement de ce secteur du fait des questions posées par la “ mise en dispositifs” de la gestion des risques sanitaires et environnementaux.

** Céline GRANJOU est Chargée de recherche au CEMAGREF (Centre d'Etudes sur le Machinisme Agricole, le Génie Rural et les Eaux et Forêts). A l'articulation entre sociologie des sciences et sociologie politique, elle travaille sur les nouvelles représentations et pratiques liées à l'intégration des questions de risque et d’environnement. 


\title{
Résumé
}

L'objet de cet article est d'éclairer la complexité et la dynamique des relations entre action publique, expertise et recherche, en s'appuyant sur l'analyse des activités d'expertise sur le domaine particulier des ESST et des prions. Le travail de recherche s'est adossé à une lecture diachronique de l'expertise dans ce domaine pour suivre la structuration conjointe d'une politique de recherche et d'une politique sanitaire. Nous caractérisons tout d'abord l'état d'incertitude des connaissances scientifique sur les prions et la lente émergence de ce domaine de recherche en France. Puis nous donnons une lecture sociologiques des pratiques de l'expertise. Nous nous attachons à montrer comment la mobilisation des chercheurs dans un comité d'experts ad-hoc sous-tend la structuration d'une politique inédite de la précaution vis-à-vis des maladies à prions, et comment l'expérience de ce dispositif par les experts eux-mêmes vaut d’être considérée dans les débats qui animent la sociologie des risques aujourd'hui.

\section{Mots clés}

Expertise scientifique - maladies à prions - Comité d’experts - risque sanitaire - précaution - action publique

\begin{abstract}
The purpose of this article is to shed light on the complexity and the dynamic of relation between public decision making, scientific expertise and research policy thanks to the analysis of expertise activities in the particular field of TSEs and Prions. Our field research consists in a longitudinal study of expertise in order to follow-up the joint structuration of a research policy and of a sanitary policy. We firstly characterise the state of uncertainty in scientific knowledge about the mechanisms and origin of those transmissible diseases and the slow emergence of this research domain in France. Then we deliver a sociological approach of expertise practices: human organizing, production of scientific advises, and resources in relation to research activities. We try to acknowledge and analyse how the mobilisation of researchers in an ad-hoc expert committee has grounded a precautionary public policy about Prions diseases, and how the experience of this setting by experts themselves has to be considered within the sociology of risk and expertise as far as debates about participation are concerned.
\end{abstract}

\section{Key-words:}

Scientific expertise - Prions diseases - expert committee - sanitary risk - precaution - public decision 


\section{Introduction}

Depuis le début de l'épidémie de “vaches folles” au Royaume-Uni au milieu des années 80, les chercheurs français travaillant sur le domaine des prions tendent à être sollicités comme experts afin d'aider à définir les orientations de la politique gouvernementale en matière de protection contre les risques liées aux maladies à prions. S'il s'agit d'abord d'une forme d'expertise d'administration ${ }^{1}$, l'action publique étant alors marquée par une juxtaposition de logiques sectorielles que l’irruption de l’hypothèse de transmissibilité en mars 1996 a rendu a posteriori patente, la crise de 1996 a généré une large réorganisation de la prise en charge publique de la sécurité sanitaire, y compris au niveau européen ${ }^{2}$. On assiste alors en France à l'émergence simultanée d'un champ de recherches - très faiblement constitué avant l'éclatement de la crise en 1996 - et d'un champ d'intervention publique sur les Encéphalopathies Spongiformes Subaiguës transmissibles (ESST) et les prions (prolifération réglementaire, mises sous embargo, action diplomatique, communication sur les risques). Dans le même temps se structure, en plusieurs étapes, l'organisation de lieux et de procédures selon lesquels les chercheurs doivent évaluer de façon collégiale les risques liés aux prions à partir des connaissances scientifiques disponibles. Après le rapport rédigé par Dominique Dormont en $1992^{3}$ et appelant, sans succès, au développement d'un Groupement d'Intérêt Scientifique (GIS) sur les ESST et les maladies à prions, l'institution en 1996 du comité interministériel sur les ESST (Ciesst ou “comité Dormont”) - regroupant missions d'évaluation des risques et d'animation de la recherche-, puis du GIS Prions fin 2000 et enfin du Comité d'Experts Spécialisés sur les ESST de l'AFSSA en 2001, scandent cette organisation progressive, offrant diverses configurations de relations entre recherche, expertise et action publique.

Il est du coup pertinent de s'adosser à une lecture diachronique de l'expertise dans ce domaine pour suivre la structuration conjointe d'une politique de recherche et d'une politique sanitaire, d'autant que ces deux types de politique s'appuient pendant quatre ans sur le même comité d'experts, le Ciesst. Reconstituer l'histoire et les

1 Au sens de Christine RESTIER-MELLERAY, “Experts et expertise : le cas de la France ”, Revue française de science politique, 40, 4, 1990, p. 540-585 ; pour un point sur les débats visant à dépasser le modèle d'expertise d’administration, voir Céline GRANJOU, “L'expertise scientifique à destination politique”, Cahiers internationaux de sociologie, 114, 2003, p. 175-183.

2 Sur la question de la constitution de l'ESB comme problème public européen voir Marc BARBIER, “ Une interprétation de la constitution de l'ESB comme problème public européen ”, Revue Internationale de Politique Comparée, Les politiques des risques en Europe, 10, 2, 2003, p. 233-246.

3 Dominique DORMONT, Les encéphalopathies subaiguës spongiformes humaines et animales : description clinique et biologique, facteurs étiologiques, conséquences sur la santé publique, et axes de recherches développées en France, Rapport au Ministre de la recherche et de l'espace Hubert Currien, (confidentiel), 1992. 
étapes de cette dynamique offre une occasion particulière d'observer les modalités de l'intervention des chercheurs dans l'action publique et le rôle qu'y joue la mobilisation des savoirs académiques pour légitimer et/ou appareiller l'action publique.

A l'heure où bien des clivages apparaissent dans la sociologie de l'expertise autour de la question de la participation des profanes, des usagers ou des citoyens $^{4}$, il nous semble important de définir le regard sociologique que nous portons sur les activités d'expertise. S'il nous semble primordial de reconnaître le rôle des Sciences Studies dans la mise à plat parfois critique d'une diversité de situations et de dispositifs d'expertise marqués par la mise en avant de l'autorité scientifique, Harry Collins et Robert Evans soulignent à juste titre l'existence d'asymétries de compétences et par conséquent d'un droit différencié des acteurs à éclairer la décision publique : le droit de participation politique de tout citoyen ne doit pas être confondu selon eux avec la capacité à contribuer à l'expertise. Considérant donc qu'une sociologie de l'expertise ne consiste pas seulement à étudier des collectifs hétérogènes pour étayer une théorisation de la participation, nous avons fait le choix ici de considérer des configurations plus “ classiques” d'expertise scientifique, mais dans une situation d'expertise qui n’est pourtant pas “habituelle” car marquée par l'émergence d'une référence accrue à la précaution. Nos travaux de recherche ont ainsi visé à comprendre les pratiques de l'expertise scientifique sur les ESST dans un tel contexte qu'elles contribuaient aussi à former : la référence à ce qui devient un standard de la décision publique en situation d'incertitude ${ }^{5}$ était en effet alors beaucoup moins constituée qu'elle ne l'est aujourd'hui, précisément du fait du rôle de précédent que jouent l’affaire du sang contaminé et les deux “ crises de la vache folle ”.

Afin d'échapper à la tentation d'une explication en terme de transition d'un modèle d'expertise confisquée par les corporatismes agro-industriels (prédominant jusqu'à l'éclatement de la crise) à un modèle d'expertise éclairée et transparente (reposant sur une nouvelle communauté de spécialistes) ${ }^{6}$, nous avons opté pour une sociologie empirique et pragmatique des activités d'expertise. Nous nous sommes attachés d'une part à la compréhension des pratiques autant que celles-ci sont observables, ou tout au moins à leurs conditions, à leurs ressources et à leurs produits ${ }^{7}$, et avons visé, à travers l'entretien sociologique, à comprendre le sens que les experts donnent

4 On renvoie ici à l'article Harry COLLINS et Rob EVANS, “The third wave of "Science Studies ” : studies of expertise and experience”, Social Studies of Science, 32, 2, 2002, p. 235-296, et aux discussions qu'il a déclenché dans la revue Social Studies of Science (voir le numéro 3 de l'année 2003) à propos du sens d'un retour à une conception normative des genres de connaissance qui définissent l'expertise scientifique.

5 On suivra un auteur comme Laurence BOY, "La nature juridique du principe de précaution ”, Natures Sciences Sociétés, 7, 3, 1999, p. 5-11 qui voit dans l'invocation de la précaution plus un standard qu'un principe de droit.

6 Nous suivons en ce sens l'orientation définie par Nicolas Dodier dans son approche de l'action collective autour de l'épidémie de Sida (Leçons politiques de l'épidémie de Sida, 2003, éditions de l'EHESS; voir aussi la communication de Nicolas Dodier au Séminaire “ Risques Collectifs et situations de crise” en mai 2002).

7 Selon une approche centrée sur les équipements de l'expertise proposée par Jean-Yves TREPOS, La 
eux-mêmes à leurs activités. Notre travail n’a donc pas consisté à porter un jugement sur l'efficacité ni sur la légitimité du dispositif, mais à cerner, par une approche compréhensive, la façon dont l'expertise fait l'objet d’une prise de conscience réflexive individuelle et collective. Nous nous attacherons ainsi à montrer comment la mobilisation des chercheurs dans un comité d'experts placé à l'articulation entre recherche et action publique sous-tend fortement la structuration d'une politique inédite de la précaution vis-à-vis des maladies à prions, et comment l'expérience de ce dispositif par les experts eux-mêmes vaut d'être considérée dans les débats qui animent la sociologie des risques aujourd'hui. Dans ce cadre, on ne réduira pas la façon dont l'expertise permet l'alliance entre recherche et action publique à une explication en terme de déterminations externes pesant sur les “ chercheurs-experts”, qui souligneraient les risques dans leurs avis parce qu'ils seraient guidés par des enjeux d'insertion professionnelle ou par l'anticipation d'une critique inspirée de l'affaire du Sang Contaminé vis-à-vis du défaut d'anticipation d'un danger pour la collectivité. Les discours et pratiques des experts témoignent en effet d’une réflexion permanente sur la façon dont ils peuvent conduire leurs activités sous l'égide d'un référentiel vécu en partie comme nouveau et perturbant, à savoir celui de la précaution.

L'objet de cet article est ainsi d'éclairer la complexité et la dynamique des relations entre action publique, expertise et recherche, en s'appuyant sur l'analyse du fonctionnement de l'expertise sur le domaine particulier des ESST et des prions. Notre démarche s’appuie sur les résultats de divers travaux formant un portefeuille de cas d'études menés successivement, dans le cadre du CRIDE ${ }^{8}$, du projet “ L'expertise du CIESST à la frontière entre recherche et décision publique ” dans le cadre du GIS Prions, ainsi que d'une thèse de doctorat ${ }^{9}$. Les matériaux recueillis sont de divers types (entretiens, archives, bases de données regroupant articles de presse, articles scientifiques...) sachant qu'il n'a en effet pas été possible d'assister aux réunions de par la réticence des experts. Ces matériaux permettent ainsi une approche diachronique et nuancée du fonctionnement de l'expertise, à travers l'analyse du travail de formulation collective de jugements tendant à définir l'action publique souhaitable par des chercheurs qui s'approprient un rôle d'expert souvent nouveau pour eux, tout en restant liés à leur institution de rattachement. Les archives du GIS Prions - que notre travail de terrain a eu pour effet de trier et d'organiser- ont tout particulièrement permis une reconstitution approfondie du type d'activités du Ciesst, qui permet de compléter les témoignages issus des entretiens ${ }^{10}$. Nous avons également bénéficié des nombreux travaux qui

sociologie de l'expertise, Paris, Presses Universitaires de France, 1996.

8 Projets du Collectif de Recherche sur l'Innovation, la Décision et l'Expertise, INRA qui ont porté sur les relations entre expertise et décision publique (projet Européen BASES, projets financés par le Programme Risques Collectifs et Situations de Crise).

9 Céline GRANJOU, “La gestion des risques entre technique et politique. Comités d'experts et dispositifs de traçabilité à partir des exemples de la vache folle et des OGM ”, thèse de sociologie soutenue à l'université Paris 5, le 18 février 2004.

10 Nous remercions le Prof. Luigi di GIAMBERARDINO secrétaire général du GIS Prions de nous avoir ouvert 
concernent de près ou de loin ce dossier, avec des approches plus centrées sur l'action collective, la décision publique, les risques collectifs ou l’expertise à finalité politique. La “ vache folle” a été ainsi depuis la crise de 1996 un dossier protubérant et en même temps insaisissable dans sa globalité tant sont nombreux les discours qui y ont trouvé une puissante métaphore pour condenser la vision prémonitoire d’Ulrich Beck au début des années 90.

Nous tenterons de montrer comment les pratiques de l'expertise scientifique à finalité politique participent à la construction des cadres ou certains diront des référentiels d’action publique en matière sanitaire, à travers la notion de pragmatique de la précaution. En d'autres termes, on se situe ici dans une fabrique non juridique du droit, quelque part en amont des cabinets et des directions générales. Il s'agit de s'attacher non pas aux jeux entre les experts et les tutelles prenant la situation d'expertise et l'avis d'expert comme un simple prétexte à des enjeux situés ailleurs, mais bien de rendre compte de ce qui a permis la production de règles en matière de sécurité sanitaire sur les ESST. Pour cela, après avoir caractérisé l'état d’incertitude des connaissances scientifique sur les prions et la lente émergence de ce domaine de recherche en France, nous passerons par plusieurs entrées dans la pratique de l'expertise : son organisation pratique, l'activité d'écriture des avis, et enfin ses ressources et ses liens avec l’activité de recherche.

\section{La lente structuration d'un domaine de recherche en France}

\section{I.1. Naissance d'un intérêt pour les ESST}

Pour bien comprendre l'investissement de recherche sur le domaine des ESST en France avec le programme de recherche lancé en 1997 puis avec le GIS Prions en 2001, il est nécessaire de resituer cet investissement dans l'arrière-plan historique de la “ science monde ” : celle-ci montre une tout autre activité que celle que connaît la recherche française à la fin des années quatre-vingt, moment où l'existence d'une nouvelle ESST chez les bovins signifie aussi la naissance d’un intérêt pour les mécanismes de la transmission de ce type de maladie et sa pathogénicité. Le domaine de recherche sur les ESST et les Prions, tel que défini par l'activité de publication scientifique identifiée par la base de données MEDline entre 1986 et 1997, montre une expansion en pallier qui suit l'épidémiologie des ESST et leur retentissement dans l’espace social (voir l’annexe 1).

l’accès à ces archives. 
Par ailleurs, différents types d'ouvrages couvrent la caractérisation de la dynamique des connaissances scientifiques de ce domaine : des travaux de popularisation rédigés par des scientifiques, des travaux d'histoire scientifique internaliste $^{11}$ et de nombreux récits journalistiques dont certains fort bien documentés ${ }^{12}$. Cette littérature permet notamment de bien mesurer l'état de tension qui préside à la nomination de S.Prusiner au prix Nobel de médecine en 1997. Cette question des tensions professionnelles liées à la dynamique professionnelle des sciences ne renvoie pas seulement aux oublis que ce genre de récompense induit au niveau des contributeurs et alliés, mais aussi à des tensions épistémiques fortes. En effet le modèle théorique que propose S.Prusiner en 1982 repose sur un renversement complet du dogme central de la biologie moléculaire proposé par F. Crick en 1958 établissant qu'il ne peut y avoir de transfert d'information d'une protéine à une autre sans acide nucléique $^{13}$.

C’est donc dans ce contexte de tension et après le scandale sur les hormones de croissance que le domaine de recherche sur les ESST et les Prions tente d'exister en terme de politique scientifique à la suite du rapport Dormont de 1992. A cette époque, deux chercheurs principalement travaillent dans ce domaine, en lien avec le laboratoire de Daniel Gajdusek (premier prix Nobel de ce domaine en 1976 avant celui de Stanley Prusiner puis de Kürt Wütricht) : Françoise Cathala, neurologue à l'Hôpital de la Salpêtrière en lien avec Paul Brown, et le

Général Louis Court en neurophysiologie comparée ${ }^{14}$. C’est ce denier qui donnera les moyens à Dominique Dormont de s’établir dans ce nouveau domaine de recherche après avoir collaboré très activement à celui du HIV, tandis que Françoise Cathala est la première à poser et instruire la question d'un lien possible entre la tremblante du mouton et la maladie de Creutzfeldt-Jakob.

\section{2 : Naissance d’une politique de recherche}

La structuration des activités de recherche par une action publique commence de façon embryonnaire avec la création du Centre National de Référence sur les ESST à Lyon au CNEVA. Par ailleurs, un projet européen dans

11 Nous avons consulté notamment C.-M. POSER, “ Notes on the history of the prion diseases ”, Part I and Part II, Clinical Neurology and Neurosurgery, 104, 2002, p.2-9 and p.77-86, ainsi que R.-M. RIDLEY and H.-F. BAKER, Fatal protein, The story of CJD, BSE and other Prion Diseases, Oxford, Oxford University Press, 1998, mais il existe bien autre contributions dont la recension mérite un travail particulier.

12 On pense ici à Richard RHODES, Festins mortels: les nouvelles maladies qui menacent l'espèce humaine, Paris, Plon, 1998.

13 C'est ce renversement que décrivent tout à fait minutieusement deux article de M.-E. KEYES, “The Prion challenge to the 'Cental Dogma' of molecular biology, 1965-1991, Part I and Part II ", Stud. Hist. Phil. Biol. \& Biomed. Sci., 30, 1, p.1-19 et 2, p.181-218, 1998.

14 La mobilisation du monde de la recherche vétérinaire sera plus tardive consécutive à l'instauration du réseau d'épidémiosurveillance de l'ESB en 1990 et à l'établissement d'un laboratoire de référence au sein du CNEVA (sui sera “absorbé" par l'AFSSA). Pour la recherche agronomique, c'est avec les sollicitations du monde de l'élevage en 1993 et du fait de l'infection du troupeau expérimental ovin de Langlade que les travaux sur la tremblante deviennent identifiable sur plusieurs fronts voir J.M. AYNAUD et F.GROSCLAUDE, L'évolution de recherches conduite à l’INRA dans le domaine des ESST, 1997, Paris, Document INRA, 57 p. 
lequel s'implique l'unité 360 de l'INSERM d'épidémiologie à la Salpétrière en lien avec Jean-Jacques Hauw (service de neuropathologie à La Salpétrtière), Jean-Louis Laplanche (Service de biochimie à Lariboisière) et Dominique Dormont (Centre national de réference des ATNC) initie un début de coordination de l'épidémiosurveillance au niveau national ${ }^{15}$.

C’est cependant la création du Ciesst et sa mission de mise à jour permanente des connaissances qui pallie l'échec de constitution d'un GIS pour la structuration d'un programme de recherche sur les ESST et les Prions. Le Ciesst met en place une animation permanente du domaine avec un programme d'appel d'offres ciblés en 1996 et un appel d'offre plus sélectif en 1997 et 1999 (voir annexe 2). La conduite de ce programme met à contribution les instituts de recherche nationaux à travers la gestion des programmes de recherche interorganismes (voir le tableau 1). Par ailleurs, avec un effet de structuration également très fort, le nombre croissant de chercheurs et de projets sur les ESST et les prions, à l'instar de ce que nous avons décrit plus globalement, conduit à des partenariats européens au travers des programmes européens FAIR, BIOTECH et BIOMED ${ }^{16}$. Enfin, après la deuxième crise de l'ESB le gouvernement débloque une enveloppe de 32 millions d'euros pour faire fonctionner le GIS “infections à prions” dès 2001. Ce GIS reprend, avec des moyens et un engagement forts des instituts de recherche partenaires, la mission de structuration de la recherche qui était celle du Ciesst. Un premier appel d'offre est lancé en mars 2001 qui se traduit par le financement de douze laboratoire de type P3 (installations de haute sécurité pourvu d'animaleries et de laboratoires confinées) et du personnel (vingt ingénieurs et techniciens). Enfin le GIS tente de structurer une présence française sur la thématique ESST dans le cadre du cinquième puis du sixième PCRD ${ }^{17}$.

\section{Le comité Dormont : composition et activités}

Le Ciesst, à la différence de bien d'autres cas ou situations d'expertise, est remarquable en ce qu'il ne repose pas sur une population d'experts professionnels ni sur un champ d'expertise constitué. Il s'agit d'un groupe d'experts aux origines institutionnelles, académiques et scientifiques variées, pris à des moments différents de leur carrière professionnelle; le statut d'expert joue un rôle généralement légitimant et gratifiant dans leur monde d'origine

15 Sur ce point de l'organisation de l'épidémiosurveillance des ESST on pourra consulter le rapport Marc BARBIER, Jacqueline ESTADES et Elisabeth REMY, Les réseaux d'épidémiosurveillance des ESST. Une lecture sociologique de leur structuration et de leur fonctionnement, ACC3 Contrat INRA B1632, INRA Grenoble, 2001.

16 Voir l'inventaire des activités de recherche réalisé en 2001 par la direction de la recherche de la commission européenne disponible en ligne http://europa.eu.int/comm/research/quality-of-life/pdf/tse-finalreport.pdf.

17 Programme Cadre de Recherche et Développement (outil de programmation de la recherche au niveau européen). 
mais par systématiquement. Qui plus est, cette variété de positions disciplinaires sur le plan scientifique renvoie à la dynamique propre d'un domaine de recherche en constitution, bien au-delà du problème ponctuel de l'apparition d'une nouvelle maladie, l’ESB. Comment un tel collectif s’est-il organisé à partir de sa composition et de ses missions officielles pour fonctionner en pratique?

\section{1 : Un comité à géométrie très variable}

S’il existe une liste de 24 experts officiellement désignés par le gouvernement en avril 1996, les listes de présence $^{18}$ aux réunions montrent plus précisément que les frontières du comité ne sont pas aussi stables qu'il pouvait y paraître, puisque, outre d'autres scientifiques que se sont adjoints les experts nommés, des représentants des instituts de recherche participent parfois, ainsi que des membres de l'administration. Plus ponctuellement, assistent aux séances des agents des administrations centrales ou des technologues du domaine de la transformation des viandes. On note ainsi que le périmètre de l’expertise n’est pas constitué qu'autour d’un fond de connaissances scientifiques : il est aussi défini par un ensemble de relations avec les institutions et avec les porte-paroles de la réalité des risques du point de vue des pratiques ${ }^{19}$.

L’étude plus poussée de la participation des divers membres du Ciesst montre un fonctionnement suivant trois “ cercles ”. Il se dessine un noyau d'environ 16 membres ayant une participation constante et forte ; un cercle de membres ayant une participation forte au début puis nettement décroissante voire nulle pour certains ; et enfin un troisième groupe comprenant des membres ayant peu participé. Cette typologie succincte, ainsi que la diminution du nombre moyen d'experts présents à chaque séance de 96 à $99^{20}$ et l'augmentation inverse du nombre de remplacements ${ }^{21}$, dessine un phénomène de démobilisation, dans lequel l'introduction de l'AFSSA dans le “jeu de l'expertise” a joué tout autant que le fait que certains membres aient considéré que le travail d'expertise scientifique de l'après-crise (à partir de 1998) avait été suffisant pour donner les moyens aux décideurs de conduire leur politique des risques. Le régime de fonctionnement, au départ très intense et dévoreur de temps pour les personnes impliquées (une séance par mois environ en 1996 et 1997), diminue à partir de1998 (7 séances en 1998 et 1999 et 5 séances en 2000).

18 Il est frappant de noter que 46 personnes distinctes apparaissent dans les listes d'experts présents aux séances. 19 On insistera pour dire que le problème de la participation se pose pour nous tout particulièrement dans l'existence, le maintien et l'attention portés à ce genre de relations aux praticiens qui permettent de ne pas constituer les tutelles - voire même aujourd'hui les agences- comme les seuls propriétaires de la définition des problèmes à traiter. Cette question nous paraît bien plus importante que celle de savoir si les profanes et les experts pensent différemment.

20 On a les chiffres suivants concernant le taux moyen de présence globale sur l'ensemble des membres du comité : $82 \%$ en $96,64 \%$ en $97,57,5 \%$ en 98 et $59 \%$ en 99.

21 On peut distinguer deux types de remplaçants : le remplaçant ponctuel (qui apparaît dans une ou deux séances) et le remplaçant attitré et quasi définitif. 


\section{2 : Des activités particulièrement hétérogènes ${ }^{22}$}

Suite à l'annonce de l'hypothèse de transmissibilité de l'ESB à l'homme est mis en place sous la tutelle de trois administrations centrales (alimentation, santé et répression des fraudes) “un comité d'experts de veille scientifique, médicale et technique sur les encéphalopathies subaiguës spongiformes transmissibles et les prions "23. Les missions officielles de ce comité pluridisciplinaire sont au nombre de trois : “la mise à jour permanente des connaissances, la fourniture d'éléments destinés à orienter nos décisions en matière sanitaire tant dans le domaine animal que vis-à-vis de la santé humaine, la proposition dans les meilleurs délais d'un programme de recherche inter-organismes ”. Le Ciesst, qui fonctionne en rendant des avis sur saisine des administrations de tutelle, est ainsi conçu comme une sorte de réservoir de connaissances mobilisables tant pour appuyer un travail administratif d'élaboration réglementaire que pour orienter la recherche.

Les compte-rendus du comité reflètent des activités finalement très variées liées à l'étendue de ses missions. La place attribuée à la gestion des appels d'offres est souvent supérieure à celle attribuée aux réponses aux saisines même s'il est difficile d'estimer le temps effectivement consacré à chaque point. Certaines réunions n'ont traité aucune saisine, ce qui indique bien que l'élaboration des avis n’est pas un travail collectif en séance plénière mais qu'il se situe ailleurs dans de petits groupes de travail (souvent par courriels), et dans des réflexions plus solitaires en lien parfois avec l'insertion scientifique propre de chacun.

Mais au-delà du travail de constitution et d'évaluation des appels d'offre du programme, une grande place semble avoir été donnée à l'échange d'informations d'actualité de divers ordres, faisant du comité une sorte de lieu de coordination et de partages d'informations sur tout ce qui pouvait concerner le domaine des ESST sur un plan scientifique mais aussi sur le plan de la gestion des risques et de la veille sanitaire

L’étude des modalités pratiques de fonctionnement du comité aboutit ainsi à la vision d'une activité particulièrement hétérogène, au sein d’un collectif à géométrie variable marqué par une implication différenciée

22 Nous ne donnons ici qu'une description succincte des activités du Ciesst, voir sinon la batterie de travaux : Pierre-Benoît JOLY, Yves Le PAPE, Elisabeth REMY, "Quand les scientifiques traquent les prions. Le fonctionnement d'un comité d'experts dans la crise de la vache folle ", Annales des Mines - Responsabilité et environnement, 9, 1998, p. 86-95 ; Jacqueline ESTADES, Marc BARBIER et Elisabeth REMY, “Les comités d'experts comme dispositif de production de confiance dans la gestion publique des risques: le cas de l'ESB ”, in François Aubert et Jean-Pierre Sylvestre (sous la dir.), Confiance et Rationalité, Paris, INRA-Editions, 2001; Marc BARBIER, Jacqueline ESTADES, and Pierre-Benoît JOLY, “ The institutionalisation of the precautionary principle in France through the Beef War", Joint Conference 4S/EASST, Vienne, 2001; Marc BARBIER et Céline GRANJOU, “ Experts are learning. A case study”, communication à la conférence EGOS, Copenhague, 2003; Jacqueline ESTADES et Elisabeth REMY, L'expertise en pratique : les risques liés à la vache folle et aux rayonnements ionisants, Paris, L'Harmattan, 2004.

23 Les citations sont tirées de la lettre de mission du 17 avril 1996 au président du Comité Dominique Dormont (archives GIS). 
des différents membres et par une certaine démobilisation à partir de 1997. L’analyse plus poussée de la pratique de l'expertise va maintenant nous mener vers l'activité de fabrication et d'écriture des avis au sein de ce collectif, qui nous permettra d'introduire l'idée d'une pragmatique de la précaution.

\section{La fabrique des avis d'expertise : une pragmatique de la précaution}

Les écrits intermédiaires des avis que nous avons pu rassembler dans les archives du comité ${ }^{24}$ permettent de reconstituer les enjeux de l'activité de fabrication des avis en réponse aux tutelles. Nous disposons en effet des versions intermédiaires de plusieurs avis ainsi que de commentaires d'experts sur ces versions, écrits qui constituent un accès privilégié au cœur de l'activité d'expertise. Bien que ces textes intermédiaires n’aient pas été conçus dans l'objectif d'affirmer expressément une traçabilité de cette activité (la conservation de ces écrits intermédiaires est très loin d’être systématique), ils peuvent être interprétés comme des écrits d'action ${ }^{25}$ dans le sens où leur production s'inscrit tout de même dans une routine de fonctionnement du comité pour cette activité $^{26}$.

\section{III.1 : Un triple horizon de sens pour l'écriture des avis}

Le comité confiait généralement la rédaction d'un premier avis à 2 ou 3 rapporteurs “ experts parmi les experts ”, et ce premier jet de l'avis était mis ensuite en circulation au sein du comité pour faire l'objet d'une discussion en séance plénière. C'est à la suite de ces discussions, dont on ne peut aujourd'hui connaître l'exacte teneur, que ce premier texte pouvait alors faire l'objet d'une nouvelle rédaction correspondant parfois à l'élargissement du cercle des rédacteurs. La métaphore de l'atelier d'écriture convient pour se faire une idée de ce travail d'écriture à “ plusieurs mains ”.

Notre travail sur les variations de ces textes a porté sur tant sur le repérage des modifications qui visent l’intelligibilité des énoncés que sur celui de l'évolution des motifs argumentaires et enfin sur l'analyse de

24 Nous avons étudié la genèse de cinq avis : la première auto-saisine du comité (avis du 9 mai 96) ; l'avis du 29 juillet 96 sur la transmission de l'ESB aux ovins et caprins ; l'avis du 27 juin 96 sur les farines de viande et d'os ; l'avis du 28 septembre 96 sur l'utilisation d'asticots comme appâts; l'avis du 30 mars 97 sur l'utilisation de viandes d'origine britanniques, importées avant la mise en place de l'embargo.

25 Sur la notion d' “ écrits d'action ”, voir Béatrice FRAENKEL, "La traçabilité, une fonction caractéristique des écrits au travail ", Connexions, 65, 1995, p.60-75.

26 Sur l'idée du travail des experts comme production de traces, voir Jacqueline ESTADES et Elisabeth REMY, “Sur les traces de l'expert ”, Nature, Science, Sociétés, 2, 2002, p 31-38. 
l'organisation interne de la logique des textes. D’un point de vue sémantique, ces modifications sont les ressources que les auteurs mobilisent pour doter les textes, qui seront rendus publics, de certaines propriétés et par la même occasion pour positionner le comité lui-même sur la scène publique.

On peut repérer dans cette fabrication des avis trois horizons de sens orientant l'écriture. Une contrainte de type instrumental correspond au fait que la fonction première de l'écriture est de produire un avis qui repose sur des connaissances scientifiques solides et qui synthétise l'articulation entre des points de vue de spécialistes. Sur ce plan, les versions initiales des avis contiennent des exposés que l’on peut qualifier de “ pédagogiques ” dans la mesure où la mise en forme d’un argument nécessite pour les rédacteurs de décrire le contexte dans lequel l’argument prend source. D’une manière générale, ces exposés font l’objet d’une “ épuration ” dans la version finale, le monde tel qu’il est étant peut-être supposé connu des tutelles. L’engagement des experts dans ce niveau d'écriture correspond à la recherche d'une justesse dans ce qu'il convient d'affirmer et de mobiliser dans un ensemble de connaissances scientifiques qui n'ont pas vocation à être des recommandations pour l'action publique.

Une deuxième contrainte que l’on peut qualifier de sociale est liée au fait que dans l'écriture se jouent également la cohésion et la pérennité du collectif : si la rédaction initiale est déléguée à quelques personnes, le texte final doit recueillir l'approbation de l'ensemble des membres, selon une règle de recherche du consensus revendiquée par le comité. Les variations ne sont donc pas le simple produit de la recherche du texte le plus juste eu égard les réserves de connaissances scientifiques disponibles, il s’agit aussi pour les experts de penser le collectif dans sa durée.

Enfin une contrainte que l'on peut qualifier de politique renvoie à la façon dont le comité tente de définir son rapport aux tutelles et à la situation d'expertise elle-même, dans un contexte où les experts anticipent l'usage que les tutelles peuvent faire du texte produit. Le travail d'écriture exprime une position du collectif vis-à-vis des enjeux de protection de la santé animale et humaine et reflète selon un engagement de la responsabilité des experts dans la définition d’un bien commun, la santé publique.

Le travail d'écriture des experts peut donc s’interpréter comme la fabrication d'un texte juste, consensuel et enfin politique. L’étude des variations entre les versions permet ainsi de mettre ainsi à distance certaines conceptions des relations entre experts et décideurs marquées par des inférences de manipulations réciproques souvent plus supposées qu’étayées. C’est en effet ce que suppose, soit le modèle technocratique de l'expertise où les experts 
organisent le monde en “ court-circuitant” le débat démocratique au nom de leurs connaissances établies, soit le modèle “ décisionniste” où les décideurs instrumentent les experts pour parvenir à leurs fins ${ }^{27}$. Notre travail montre que nous sommes beaucoup plus proches d'un modèle pragmatique de l'expertise, les experts cherchant dans la fabrication des avis à frayer un chemin entre ces deux modèles. Si le spectaculaire retournement du texte final de l'avis sur les asticots (septembre 1997) montre comment les experts se sont désengagés d'une posture d’alerte et ont volontairement évité une formulation des risques qui aurait pu être réappropriée dans un discours de justification de l'action sécuritaire des pouvoirs publics, d'autres genèses d'avis témoignent du souci de ne pas émettre de recommandations qui dicteraient aux politiques leur décision. C’est le cas de la genèse de l'avis sur les farines de viande et d’os (aboutissant, en juin 1996, à des recommandations de procédures de sécurisation et non pas à l'interdiction initialement envisagée) comme de l'avis sur la filière ovine (recommandant la réalisation de recherches plutôt que les mesures d’abord suggérées d’embargo sur les ovins anglais, juillet 1996). Ces genèses montrent d'une façon générale combien la formulation du risque est informée par un refus de trop s’engager dans un processus de décision politique, dans la mesure où bien souvent, le texte final tempère les hypothèses présentées comme plausibles dans les versions précédentes et apparaît comme moins engagé que les versions précédentes, plus “ précautionneuses ”.

L’étude des variations des versions successives d'un même avis souligne à tout le moins la réflexion permanente des experts sur leur propre rôle et témoigne des subtiles nuances de leur positionnement dans le processus d'action publique. Dans ces diverses formulations et reformulations du risque se joue un équilibre qu'il est difficile de démêler entre la considération des limites des indices scientifiques et un parti-pris pour une précaution mesurée de la part du comité : dans la genèse de l’avis sur la transmission de l’ESB aux ovins, la simultanéité de commentaires portant sur le désengagement d'une position trop stricte de prescripteur et de commentaires portant sur la qualification du degré de plausibilité de l’hypothèse de transmissibilité montre qu’il peut être irréaliste de vouloir distinguer l'antériorité d'une argumentation d’ordre factuel - recherchant la “justesse” du discours - par rapport à une argumentation fondée sur le refus d'un engagement dans des recommandations trop claires et trop coûteuses sur un plan politique. C’est cette activité continue des experts de rééquilibrage d’une posture intermédiaire entre l'alerte sur les risques au nom d’une philosophie de la précaution et le désengagement politique que nous avons qualifiée de “pragmatique de la précaution ”.

27 On renvoie ici à Jürgen HABERMAS, La science et la technique comme idéologie, Paris, Gallimard, 1978, p.105-107. 


\section{III.2 : La précaution entre bon sens, “philosophie” et recomposition du statut d'expert scientifique}

La participation de Dominique Dormont avec Marie-Angèle Hermitte à la rédaction d'une des annexes du rapport Le Principe de Précaution ${ }^{28}$ est pour nous symptomatique de ce qui a pu être présenté par les experts eux-mêmes comme une “philosophie de la précaution ” guidant les jugements des experts, tout comme le sont les oppositions de style qui ont pu exister entre le Ciesst et le Comité Scientifique Européen. Il est intéressant de tenter de cerner plus précisément quels ont pu être le contenu et les fonctions de la référence à la précaution dans la pratique du comité.

Une référence explicite à la précaution est faite dans $40 \%$ des avis du Ciesst (voir annexe 3) ; l'examen du contenu de ces références montre qu'il ne s'agit pas d'une invocation du principe de précaution (à part notamment dans le premier avis en mai 1996, qui recommande des mesures de protection sans attendre la certitude de la transmission à l'homme) mais la plupart du temps d'un appel à l'anticipation et à la prudence - au point que la " précaution ” soit parfois peu distincte du “ bon sens ” invoqué dans certains avis. Le témoignage des entretiens semble toutefois indiquer une référence plus complexe à la précaution dans les débats et l'élaboration d’un jugement. Plusieurs membres du comité décrivent des expériences mentales où ils suspendent un mode de jugement considéré comme rationnel au profit de raisonnements du type "est-ce que je ferais manger cela à mes enfants ? ”. Un autre exemple d'expérience mentale, plus collective, a pu être l'invocation des enfants touchés par l'hormone de croissance à partir de l'expérience de certains experts cliniciens, confrontés à un face à face avec les malades :

“Au comité, je leur ai dit : " quand vous avez en face de vous un gamin qui est en train de mourir et qui vous dit 'toi, tu ne trouveras jamais de quoi je meurs parce que manifestement tu ne sais pas ce que c'est !' Vous pouvez toujours me dire que ce n'est pas l'hormone de croissance.... (un membre du Ciesst).

La façon dont les experts parlent de leurs activités dans l'entretien sociologique ou publiquement propose globalement un curieux mélange d'assertions - qui vont de la revendication d'un discours "purement"

28 Voir Marie-Angèle HERMITTE et Dominique DORMONT, Annexe 3 : “Propositions pour le principe de précaution à la lumière de l'affaire de la vache folle ", in Philippe KOURILSKY et Geneviève VINEY, Le principe de précaution. Rapport au Premier ministre, Paris, Ed. Odile Jacob et la Documentation française, 2002, p. 341- 386. Cette contribution sonne comme le prolongement casuistique de thèses défendues par MarieAngèle HERMITTE et d'une communication de Dominique DORMONT sur son expérience (voir "La recherche agronomique européenne dans le monde du $21^{\circ}$ siècle. Quelle innovation pour l'alimentation, l'agriculture et le cadre de vie ?”, Colloque du cinquantenaire de l'INRA, Paris, Ed. INRA, 1997), l'idée force étant qu'au-delà de l'encadrement du travail d'expertise, la question de l'engagement du collectif d'experts est posés au niveau d'un droit de regard sur la justesse des décisions prises. 
scientifique à la relation des expériences mentales évoquées et à la référence à l'“ honnêteté ” de l'expert. Ces discours renvoient selon nous à autant de figures d’engagements différenciées dans un processus décisionnel, que confirment l'analyse du positionnement du comité dans le travail d’écriture comme l'étude des motifs textuels présents dans les avis (voir annexe 4). La description donnée par Dominique Dormont du rôle d'expert résume bien cette coexistence d'une pluralité de modes d'engagement d’un même expert et le balancement continuel entre les divers registres, du scientifique “pur” au lanceur d’alerte ${ }^{29}$ en passant par le co-décideur politique : “Enfin l'expert ne doit jamais quitter son rôle d'expert scientifique. Il n'est pas le conseiller du politique, il est seulement là pour donner un avis de scientifique. (...) Il appartient aux experts de ne jamais oublier l'indispensable travail de 'look back', c'est à dire de réflexion permanente sur les décisions qui ont été prises. (...) L'expert doit également veiller à ce que sa communication, , puisqu'il ne peut pas s'y soustraire, ne se juxtapose pas avec celle de la puissance publique qui seule décide. (...) A la confusion chacun risquerait de perdre son âme. En revanche si un comité d'experts estime qu'une décision prise, politique ou administrative, va à l'encontre de la santé publique, il a le devoir de communiquer sur ce point "”.

Les experts eux-mêmes, dans les entretiens, se qualifient voire se cataloguent comme représentant non seulement telle ou telle discipline, mais également telle ou telle sensibilité, telle ou telle proximité avec les objets et situations expertisées. Certains se revendiquent chercheurs fondamentalistes, et à ce titre un certain nombre d’entre eux ont rapidement estimé que les activités du comité étaient trop éloignées de leurs compétences (citant par exemple les débats sur “ la manière de découper un bœuf”!); d’autres se revendiquent au contraire expertsterrain ou experts cliniciens, qu’il s'agisse des médecins, porteurs d’une sensibilité spécifique aux aspects humains de la maladie comme le revendique explicitement l’expert cité plus haut (“je me suis senti là pour défendre un peu la dimension humaine du truc " conclut-il), ou des vétérinaires, familiers des réalités des filières d'élevage et porteurs d'une vision parfois plus économique des mesures de gestion du risque ${ }^{30}$. Ces différentes catégories semblent avoir été véritablement appropriées et actualisées dans le collectif, du fait notamment du rôle du président animateur du débat, attentif à l'expression des diverses sensibilités ( et les sollicitant au besoin...).

29 Sur la notion de lanceur d'alerte voir Francis CHATEAURAYNAUD et Didier TORNY, Les sombres précurseurs. Une sociologie pragmatique de l'alerte et du risque, Paris, Editions de l'EHESS, 1999.

$30 \mathrm{La}$ citation suivante d'un membre du Ciesst le montre très bien : "ce qui me motive, c'est d'apporter un éclairage de praticien de la tremblante, de quelqu'un qui ne pratique pas uniquement sou l'angle de l'accumulation des connaissances scientifiques, mais aussi sous celui de la mise en œuvre des mesures proposées. Je prends l'exemple du dernier projet de police sanitaire : je ne le lirai pas de la même manière qu'un collègue biochimiste. Lui le lira et dire : “ bon, pourquoi pas ? ”, moi je me dirai : “ si on fait ça, on aura ça etc. " ". 


\section{IV : La pragmatique de la précaution à l'épreuve des relations expertise / recherche}

S’il semble clair que les experts du Ciesst aient pu contribuer à la construction d’un référentiel inédit d'action publique de par le travail réflexif, reflété dans leurs avis ${ }^{31}$, que nous avons qualifiée de “ pragmatique de la précaution ”, que devient cette analyse lorsqu'on observe le devenir de l'expertise sur les ESST au sein du nouveau dispositif que constitue l’AFSSA ? Le nouveau comité, formé de 25 membres, comprend 12 membres de l'ancien Ciesst, dont le nouveau président notamment, qui revendique la pratique d’une “ précaution à niveau constant”. Illustrant la continuité de cette “philosophie de la précaution”, le nouveau comité souligne et rappelle fréquemment la gravité de l’hypothèse de transmission de l'ESB aux ovins (“ ESB ovine ”) à l’attention des pouvoirs publics, réticents à prendre des mesures. Le contexte d'expertise est cependant tout différent : tandis que l'Agence, s’intercalant entre le comité et les récepteurs de l'avis, remplace le face à face experts/décideurs par un jeu à trois, les relations entre expertise et recherche ont également fortement évolué. Nous voudrions ici mettre en évidence la façon dont les deux dispositifs successifs d'expertise (Ciesst et comité AFSSA) renvoient à des configurations très différenciées du point de vue des ressources scientifiques de l'activité d'expertise. Cette partie permettra notamment de montrer que la référence à la précaution dans le nouveau comité s’appuie désormais sur des activités expérimentales et calculatoires totalement inexistantes au Ciesst, où le manque crucial de données a pu favoriser une pratique particulièrement discursive de l'expertise.

\section{IV.1 : Le comité AFSSA : un nouveau contexte institutionnel, organisationnel et scientifique}

L'AFSSA, explicitement créée en réaction au modèle d'expertise d'administration accusé de favoriser les intérêts économiques au détriment des intérêts de santé publique, vise à “déconfisquer” l'expertise ${ }^{32}$. Saisie obligatoirement sur tout projet de réglementation concernant l'alimentation et dotée d'une capacité d'auto-saisine, l'agence se fonde sur la consultation de comités scientifiques spécialisés ainsi que sur les travaux de laboratoires internes, et expérimente de nouvelles procédures de publicité du fonctionnement et des résultats de ses travaux.

31 Il faut noter que la quasi-totalité des recommandations émises dans les avis du Ciesst ont été suivies par les pouvoirs publics, qui en avaient même fait une ligne de conduite explicite.

32 L'Agence est créée par la loi du 1er juillet 1998, puis le décret du 26 mars 1999 organise son fonctionnement. Sur ses principes de fonctionnement, voir Martin HIRSCH, “L’expertise indépendante dans un établissement public : l'exemple de l'Agence Française de sécurité Sanitaire et Alimentaire ”, Etudes et documents du Conseil d'Etat, 52, p. 427-440. 
L’expertise scientifique sur les ESST et les prions s’est donc déroulée successivement dans trois configurations organisationnelles : le Ciesst seul, le Ciesst avec l'AFSSA et l’AFSSA avec son propre comité (figure 1).

\section{Figure 1 :}

Chronique des avis d'expertise rendu par les comités d'experts sur les ESST et par l'AFSSA (1996-2002)

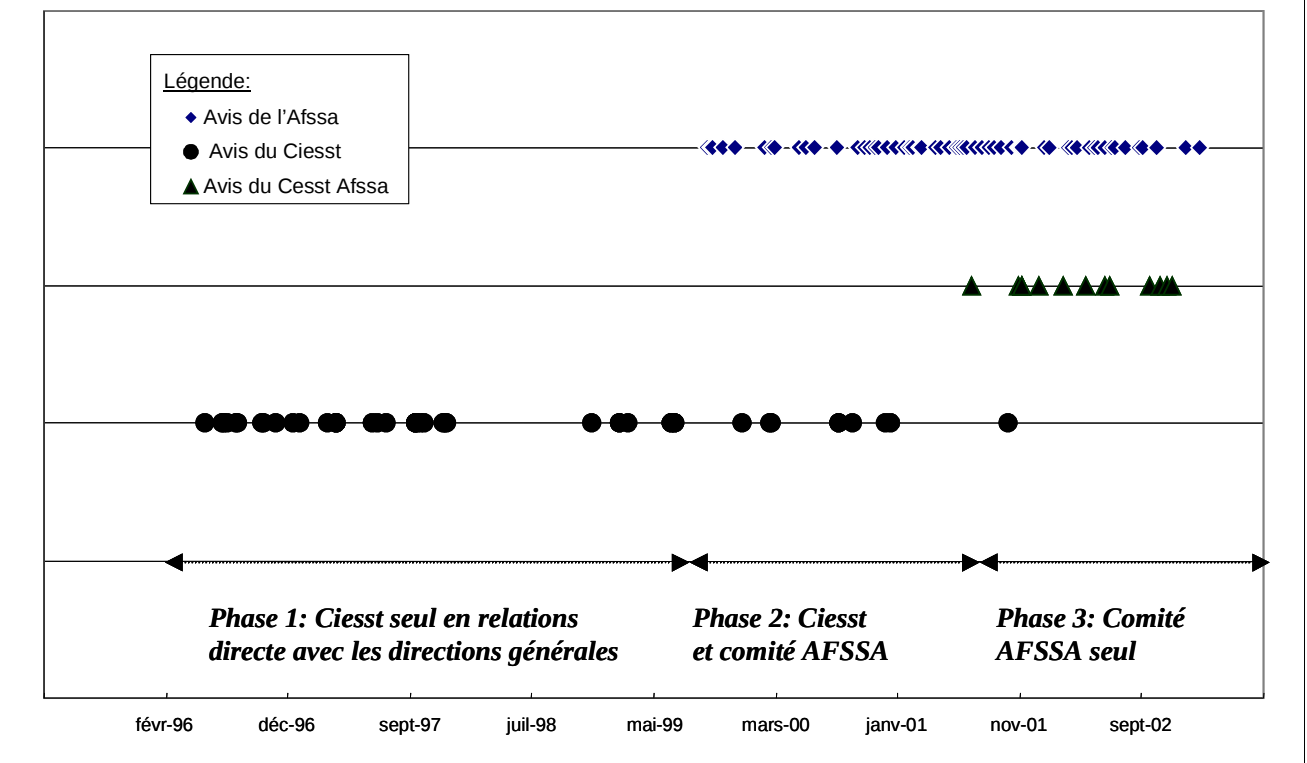

La présence de l'AFSSA induit d'abord un nouveau partage des tâches dans le traitement des saisines entre membres du comité et personnel permanent de l’Agence (secrétariat scientifique, Directeur Général). Un premier texte - le rapport - est produit par des membres du comité nommés rapporteurs de la saisine ; ce rapport est discuté collectivement en séance. Le secrétariat rédige alors un premier avis - l’avis du comité - à partir de ce rapport et des notes prises lors du débat du comité. Cet avis est soumis au président du comité pour validation ou correction éventuelle. Le secrétariat rédige ensuite l'avis final - avis de l’AFSSA - qui intègre généralement l'avis du comité de façon non littérale, et sera transmis au Directeur Général pour signature. Cette procédure correspond à une répartition des tâches entre le personnel permanent, chargé d'un travail de rédaction, et les experts, chargés d'un rôle de validation et de critique des textes qui leur sont soumis. Cette répartition vise à laisser aux experts le seul "challenge scientifique de haut niveau" 33 . En pratique, le travail d'instruction effectué par les rapporteurs présage de façon très nette le contenu de l’avis final.

Parallèlement à cette division du travail, de nombreuses ressources, tant documentaires qu’organisationnelles, sont désormais à la disposition des experts pour instruire leur jugement. La disponibilité non seulement de

33 Citation d'un entretien avec un responsable de l'AFSSA. 
données nouvelles (notamment sur l'infectiosité des différents tissus et sur la répartition de l’ESB parmi les différentes catégories de bovins), mais aussi de moyens inédits au nombre desquels les tests de dépistage de l’ESB, qui permettent de savoir en quelques jours au lieu de quelques mois si un animal est contaminé, si tel tissu ou support est infectieux, si telle voie d'inoculation est efficace, va considérablement modifier le travail d'expertise.

\section{IV.2 : D’un rapport programmatique à la recherche à un rapport d'utilisateur finalisé}

Un indice très net de la modification induite par le comité Afssa dans le style d’expertise est donné par la simple observation de la présentation formelle des avis. Ceux du Ciesst, généralement d'une ou deux pages voire même moins à ses débuts, sont la plupart du temps exempts de toute référence bibliographique. Ceux du nouveau comité font entre 5 et 10 pages pour la plupart, incluant plusieurs dizaines de références bibliographiques et parfois de longues annexes techniques; cette longueur est notamment due au compte-rendu, à l’intérieur des avis, des démarches expérimentales et des raisonnements suivis.

Cette structure des avis reflète l'utilisation des nouvelles ressources scientifiques, techniques et organisationnelles à la disposition du comité. La possibilité d’organiser des groupes de travail de longue durée sur un thème, de même que celle de faire réaliser certaines expériences au sein des laboratoires AFSSA, permettent l'obtention de nouvelles données ad hoc permettant d’instruire et d'argumenter la réponse à la saisine : on peut penser aux expérimentations sur l’infectiosité du muscle ou sur les effluents d’abattoir. Surtout, les nouvelles données épidémiologiques sur la répartition de la maladie vont être mises à profit par les experts épidémiologistes du nouveau comité. Un groupe de travail épidémiologie est constitué dès le début du fonctionnement du comité, et aura un rôle déterminant dans la fabrication des avis concernant les levées d'embargo (en Suisse, au Portugal, en France) ainsi que dans les avis concernant l'abandon de l'abattage total des troupeaux dont un animal est atteint d'ESB.

La référence à la précaution dans la pratique de l'expertise peut alors être instrumentée par le recours à ces outils, porteurs d'objectivation. A l'horizon de l'instruction des avis se dessine du coup une discrète compétition disciplinaire entre praticiens de telle ou telle technique, chacun étant plus enclin à la confiance dans son propre outil et objet de recherche, comme le montrent des débats récurrents sur la fiabilité et la pertinence du dépistage, ou encore certaines critiques de la part des non-statisticiens à l'égard des subtilités des raisonnements 
épidémiologiques. L'épidémiologie permet en effet de calculer des sur-risques associés à différents scénarios (importation ou maintien d'un embargo, abattage ou consommation de telle catégorie d'animaux...), fondant ainsi d'une manière inédite la pratique de la précaution sur des chiffres : l'avis sur le passage à un abattage partiel des troupeaux se fonde par exemple sur un calcul complexe de probabilités aboutissant à un chiffre dérisoire. Certes, le recours au calcul de sur-risque ne résout pas tout, comme le montre bien l'exemple de l'avis sur la levée de l'embargo français sur les viandes britanniques : le groupe de travail épidémiologie, ayant calculé un sur-risque lié à la réouverture des frontières compris entre 2 et 20, refuse d'en tirer aucune conclusion et c'est l'avis final de l'AFSSA, recourant à d'autres arguments, qui conclut que l'importation “ ne serait pas de nature à remettre en cause le niveau de sécurité actuellement garanti au consommateur en France \$34.

Au total, un glissement semble bien s'être opéré entre le rapport programmatique entretenu par le Ciesst à la recherche, rapport où la carence de savoirs académiques conduisait les experts à élaborer simultanément des indices de vraisemblance et un horizon normatif de jugement tout en étant simultanément prescripteur d’axes de recherches, à un rapport d'utilisateur finalisé, où l'expertise exploite les techniques et données issues des recherches antérieures pour ses propres travaux finalisés d'instruction des saisines, tandis que la recherche se développe à distance de l'arène de l'expertise. Le témoignage de plusieurs experts passés du Ciesst au nouveau comité suggère alors la façon dont peuvent être vécus les rapports entre recherche, expertise et action publique, à partir du moment où ceux-ci se sont trouvés moins liés dans une difficile et toujours remise en cause “pragmatique de la précaution ”, alliant débat d’idées et questionnement scientifique :

“ On a peut-être plus de services rendus par rapport à la société parce qu’on essaie de quantifier les choses, mais du coup, il faut bien avouer que c'est degrés de liberté en moins, au niveau du débat et des questions scientifiques... ” (un membre du nouveau comité)

\section{Conclusion :}

Contrairement à une vision commune de l'expert comme homme de savoir accompli (et donc saturé de savoirs...), ce travail ouvre des perspectives pour établir que l'activité d'expertise collective suppose certains apprentissages. Là où les experts sont établis, à travers leur nomination, comme des personnes-ressources ayant

34 citation de l'avis de l'AFSSA du 19 septembre 2002. 
un savoir étendu sur un domaine, on montre qu'une expertise collective pluridisciplinaire comme celle du Ciesst suppose que les experts apprennent. Ils apprennent non seulement à mobiliser leurs compétences dans une arène inédite, à “ être expert” dans un collectif dont les frontières et les fonctions peuvent être bien plus étendues que ne laissent supposer sa nomination officielle, mais également à concevoir la dimension politique des relations entre ce collectif et les tutelles, ou entre ce collectif et les différents mondes disciplinaires de la recherche sur les $\operatorname{ESST}^{35}$.

Une approche pragmatique de l'expertise permet ainsi, loin des conceptions globalisantes voire normatives de l'expertise, de souligner la circulation des experts entre différents codes, différents positionnements dans le processus d'action publique, ainsi que l'existence de différentes figures d'expert appropriées et actualisées dans le collectif. Le souci de faire varier la nature des engagements des personnes semble non seulement bien présent chez les individus et dans certaines phases de discussion, mais qui plus est constitutif d'une sorte de microculture de la précaution, loin d’un modèle déterminé et systématique d’évaluation. Au nombre de ces figures différenciées de l'engagement de l'expert, il faut souligner les liens souvent faibles et discontinus avec des personnes ressources dans les administrations centrales, le monde vétérinaire et plus généralement avec les représentants de certains mondes professionnels susceptibles de renseigner les experts sur la réalité de certaines pratiques impliquées dans la problématisation des risques. Cette figure, marquée par l'extension de l'expertise vers le “monde réel ”, montre combien la séparation entre évaluation et gestion des risques n’existe pas nécessairement préalablement au processus ${ }^{36}$.

Ces remarques nous amènent à revenir sur le clivage déjà évoqué au sein des Sciences Studies concernant le problème de la participation des non experts à l'expertise, qui nous semble porteur d'une difficulté à penser, d'un point de vue sociologique, les changements de référentiel liés à l’introduction d’un standard de précaution. Si la précaution entraîne effectivement une nouvelle façon de concevoir l'extension de l'expertise, nous pensons qu’il y a aussi des formes d'évolution plus ténues que celles des dispositifs participatifs et qui renvoient justement au travail de conceptualisation que les experts peuvent faire eux-mêmes à l'égard de leurs propres engagements dans des manières variables d'être expert. Cela nous semble tout à fait caractéristique d'un style propre au Ciesst, largement dû à son président, Dominique DORMONT, dont la disparition soudaine ampute très certainement la réflexion. Notre travail pointe une question qui est celle de savoir si la rationalisation des activités d'expertises au sein des systèmes d'Agences offre ce même genre de possibilité pour construire des 35 Nous avons étayé cette approche par l’apprentissage dans Marc BARBIER et Céline GRANJOU, “ Experts are learning. A case study ”, communication au colloque EGOS, Copenhague, 2003.

36 Voir Pierre-Benoît JOLY et Marc BARBIER, “Que faire des désaccords entre comités d'experts?”, Risques, Les cahiers de l'assurance, 47, 2001, p.87-94 
réponses face à l'apparition de nouveaux risques et face à l’inertie organisationnelle qui caractérise les systèmes d'action publique sectorisée ${ }^{37}$.

\section{Pour en savoir plus}

Ulrich BECK, Risk society, Towards a New Modernity, London, Sage Publication, 1992.

Michel CALLON, Pierre LASCOUMES et Yannick BARTHE, Agir dans un monde incertain. Essai sur la démocratie technique, Paris, Le Seuil, 2001, .

Frédéric CHAUVAUD avec la collaboration de Laurence DUMOULIN, Experts et expertise judiciaire :France, XIXe et XXe siècles, Rennes, Presses universitaires de Rennes, 2003.

Olivier GODARD (Dir.), Le principe de précaution dans la conduite des affaires humaines, Editions de la maison des Sciences de l'Homme, Paris, Editions INRA, 1997.

Marie-Angèle HERMITTE, Le sang et le droit. Essai sur la transfusion sanguine, Paris, Ed. du Seuil, 1996.

Bruno LATOUR, Politiques de la nature. Comment faire entrer les sciences en démocratie, Paris, La Découverte, 1999.

Dominique MEMMI, Les Gardiens du corps. Dix ans de magistère bioéthique, Paris, Editions de l'EHESS, 1996.

Philippe ROQUEPLO, Entre savoir et décision, l'expertise scientifique, INRA Editions, 1996.

Michel SETBON, Risques, sécurité sanitaire et processus de décision, Paris, Elsevier, Coll. Médecine des risques, 2004.

Max WEBER, Le savant et le politique, (Introduction par R.Aron), Paris, Plon 10/18, 1959.

\section{Annexe Etude bibliométrique du domaine de recherche ESST et Prions}

Dans le cadre d'un travail mobilisant des techniques des sciences de l'information appliquées aux bases de

37 La recherche qui permet à cet article d'exister à été financée dans le cadre d'un projet du GIS prions (appel d'offre 2001) intitulé "L'expertise du CIESST à la frontière entre recherche et décision publique ", sous la responsabilité scientifique de Marc BARBIER. 
données Medline et SCI, nous avons constitué des corpus de notices bibliographiques des articles du domaine de recherche sur les ESST et les Prions afin de conduire des analyses quantitatives de sa dynamique ${ }^{38}$ grâce notamment à une plateforme de fouille de texte et d'extraction de motif temporels ${ }^{39}$. L'activité de publication scientifique identifiée par la base de données Medline entre 1986 et 1997, montre une expansion en pallier qui suit l'épidémiologie des ESST et leur retentissement dans l'espace social : environ 0,46 \% des articles de la base par an entre 1986 et 1989 sont consacrés à ces thématiques, puis 0.84\% par an de 1990 à 1995 (période d'épizootie d’ESB au Royaume-Uni) et enfin 1.6\% en 1996 et en 1997 (crise de la vache folle qui fait suite à l'apparition des cas de nouveaux variants de la maladies de CJD chez des adolescents et de l'existence d'un nouvelle ESST chez l'homme).

Par ailleurs, une étude massive de la base de données Medline avec l'application BELUGA montre l'enrichissement croissant des champs mots-clés indexant le contenu scientifique des articles. On note à la fois la croissance continue des termes invariants d'une période à l'autre depuis 1975, et, d'une année sur l'autre, l'apparition de nouveaux termes qui s'intensifie nettement au début des années 80 puis au début des années 90 (figure a).

Enfin, en s'appuyant sur un traitement scientométrique de la co-publication (Base SCI 1986-1997) on peut attester de la dynamique professionnelle de ce domaine et montrer un accroissement du nombre de réseaux d'auteurs co-publiant à partir de 1990 et une structuration du champ autour de quelques auteurs importants (figure b). L’étude fine de la composition des réseaux de co-publications permet de suivre des recompositions des relations de coopérations ou de mise en circulation de matériel expérimental (souris transgéniques, anticorps, souches) avec une polarisation forte sur les équipes américaines puis à partir du début des années 1990 des équipes européennes au Royaume-Uni, en Suisse et en France, par exemple autour des travaux de D.Dormont, de J.L.Laplanche et de J.J.Hauw

38 Marie-Thérèse MAUNOURY et al., “ Observer la science en action ”, Médecine/Sciences, nº4, 1999, p. 577582, on déjà montrer avec des analyses des références l'évolution et la convergence des concepts clés de la théorie du prion et ceux de l'hérédité non mendélienne. Voir également Marc BARBIER et Marie-Angèle DE LOOZE M.A., "A scientometric description of the evolution of the TSEs field. Networks of authors and research themes in the MEDLINE Database. Poster presented at the International Symposium 'Characterization and Diagnosis of Prion Diseases in Animals and Man', Tübingen, Germany, September 23rd-25 1999.

39 L'application en développement est présentée dans Nicolas TURENNE et Marc BARBIER M., “ BELUGA : un outil pour l'analyse dynamique des connaissances de la littérature scientifique d'un domaine. Première application au cas des maladies à prions ", In HEBRAIL G. and LEBART L. (Eds.), Proceedings of Extraction et Gestion de Connaissances, Clermont-Ferrand, France, 2004, 18 p. L'application est accessible sur http://www-mig.jouy.inra.fr/ turenne/soft.html 
Figure a.

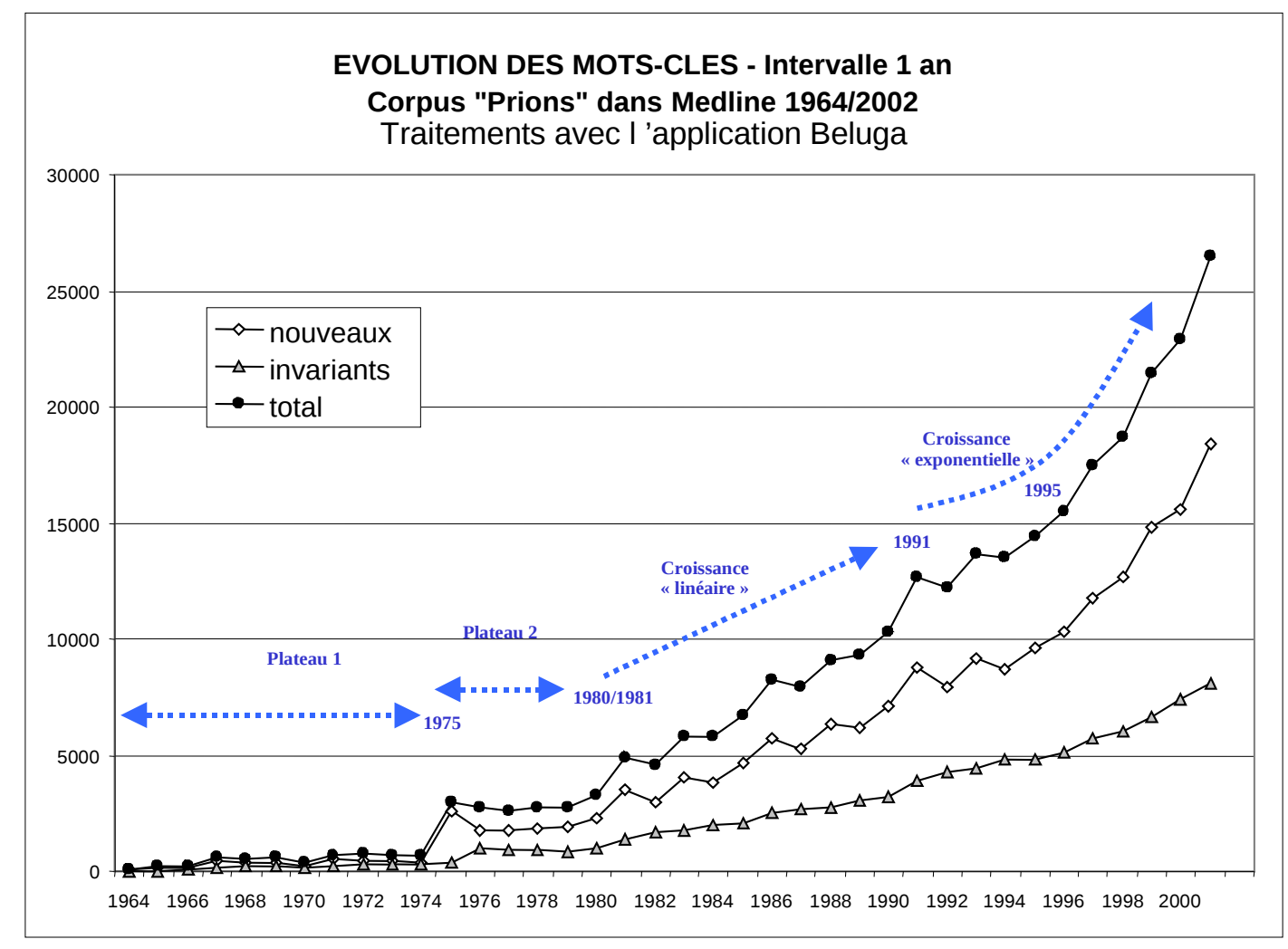

Figure b.

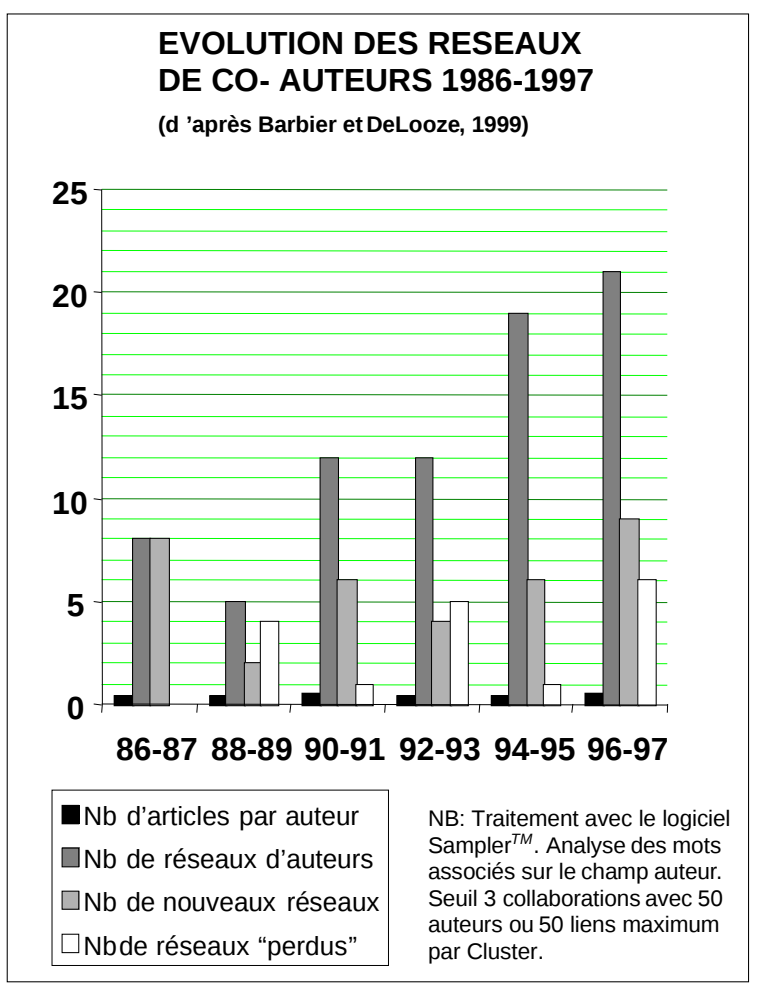


